\title{
Recent and forthcoming ONS releases
}

\section{Recent releases}

27 August

Births and Deaths 2008 registered in England and Wales (final)

www.statistics.gov.uk/statbase/product.asp?vlnk=14408

\section{August}

Internal migration estimates, mid-2007-08

www.statistics.gov.uk/statbase/product.asp?vlnk=7070

\section{August}

Mid-2008 population estimates

www.statistics.gov.uk/statbase/product.asp?vlnk=15106

\section{August}

Migration quarterly report, quarter 22009

www.statistics.gov.uk/statbase/product.asp?vInk=15230

27 August

Parent's country of birth, 2008

www.statistics.gov.uk/statbase/product.asp?vlnk=15147

\section{September}

Population estimates of the very elderly, mid-2008 and revised mid-2002-07 www.statistics.gov.uk/statbase/product.asp?vlnk=15003

\section{Forthcoming releases}

\section{September}

Quarterly population estimates (experimental), quarter 22009 and

revised quarter 32007 - quarter 12009

www.statistics.gov.uk/statbase/product.asp?vInk=13253

\section{September}

Mid-2008 household estimates, quarter 22009 and revised quarter 32007 quarter 12009

www.statistics.gov.uk/statbase/product.asp?vlnk=10755

1 October

Primary Care Organisation population estimates, mid-2008

www.statistics.gov.uk/statbase/product.asp?vlnk=15106

\section{October}

Ageing Times, new publication to coincide with International Older Persons Day

www.statistics.gov.uk/focuson/olderpeople/

\section{October}

Quarterly population estimates for English Government Office Regions and Wales, quarter 22009 and revised quarter 32007 - quarter 12009

www.statistics.gov.uk/statbase/product.asp?vlnk=13253

8 October

Mid-2007 short-term migration estimates for local authorities in England and

Wales

www.statistics.gov.uk/statbase/product.asp?vInk=15148
21 October

Life expectancy at birth and at 65 by local areas in the United Kingdom,

2006-08

www.statistics.gov.uk/statbase/product.asp?vlnk=8841

\section{October}

National interim life tables, 2006-08

www.statistics.gov.uk/statbase/product.asp?vlnk=14459

\section{October}

2008-based national population projections

www.statistics.gov.uk/statbase/product.asp?vlnk=8519

21 October

2008-based period and cohort life expectancy tables

www.statistics.gov.uk/statbase/product.asp?vlnk=15098

29 October

Mortality statistics: deaths registered in 2008 (DR)

www.statistics.gov.uk/statbase/product.asp?vlnk=15096

18 November

National population projections 2008-based additional variant population projections

www.statistics.gov.uk/statbase/product.asp?vlnk=8519

19 November

Lower Layer Super Output Area and Middle Layer Super Output Area population projections, mid-2008

www.statistics.gov.uk/statbase/product.asp?vlnk=14357

26 November

Migration Statistics quarterly report, quarter 32009

www.statistics.gov.uk/statbase/product.asp?vlnk $=15230$

26 November

Migration Statistics Long-term International Migration 2008

www.statistics.gov.uk/statbase/product.asp?vlnk=507

27 November

Quarterly Population Estimates (experimental), Third quarter 2009 www.statistics.gov.uk/statbase/Product.asp?vlnk=13523

27 November

Marital status estimates for England and Wales, mid 2008 www.statistics.gov.uk/statbase/Product.asp?vlnk=15107

For further information, contact the ONS Customer Contact Centre 0845601 3034, email info@statistics.gsi.gov.uk 